\title{
Mental Health Problems Experienced by HIV Positive Adolescents; A Case of Choma District, Zambia
}

\author{
Kestone Lyambai*, Lonia Mwape \\ School of Nursing Sciences, University of Zambia, Lusaka, Zambia \\ Email: *kestonelyambai@gmail.com
}

How to cite this paper: Lyambai, K. and Mwape, L. (2018) Mental Health Problems Experienced by HIV Positive Adolescents; A Case of Choma District, Zambia. Open Journal of Psychiatry, 8, 97-114. https://doi.org/10.4236/ojpsych.2018.82009

Received: January 21, 2018

Accepted: March 25, 2018

Published: March 28, 2018

Copyright $\odot 2018$ by authors and Scientific Research Publishing Inc. This work is licensed under the Creative Commons Attribution-NonCommercial International License (CC BY-NC 4.0).

http://creativecommons.org/licenses/by-nc/4.0/ cc) (7) (8) Open Access

\begin{abstract}
Several scholars have reported high mental health problems among adolescents with Human Immunodeficiency Virus (HIV) globally. However, little is known about the mental health of this peculiar group of young people in Choma district. This study aimed to determine the mental health problems experienced by HIV positive adolescents in Choma District. Using the Strengths and Difficulties Questionnaire (SDQ), data were obtained from 103 HIV positive adolescents receiving treatment at Choma General Hospital plus a corresponding number of parents/care givers. Information about mental health services was obtained qualitatively from 10 nurses working in the mental health unit and the Antiretroviral (ART) clinic. The results showed that more than three quarters of the adolescents had mental health problems. Of this number, 57.3 percent had multiple mental health problems: predominantly emotional and peer problems. The study also found a statistically significant relationship between level of stigma and presence of mental health problems $\left(\mathrm{X}^{2}=1.123, \mathrm{P}=0.003\right)$. In conclusion, the study demonstrated that multiple mental health problems particularly emotional and peer problems are prevalent among adolescents with HIV/AIDS yet are most often undiagnosed. In addition, adolescents with high levels of internalized stigma are more likely to have multiple mental health problems. The findings suggest the need to integrate psychiatric services into routine care of HIV infected adolescents, routine screening for mental health problems and provision of continued professional development among nurses managing these patients.
\end{abstract}

\section{Keywords}

Mental Health Problems, Experienced, Adolescents, HIV Positive 


\section{Introduction}

Mental health problems make a substantial contribution to the burden of disease worldwide. According to World Health Organization (WHO), mental disorders account for an estimated 12.3 percent of the global burden of the disease [1]. It is further estimated that, neuropsychiatric conditions account for up to 15 percent of all disability-adjusted life-years, and up to 30 percent of those attributable to non-communicable diseases. In South Africa, the Department of Health estimates that one out of four South Africans has or is affected by mental illness, but only 10 - 15 percent get help for their mental illness [2], while in Zambia, hospital based figures show a prevalence between 1.8 and 3.61 per 10,000 population for acute psychotic states and schizophrenia respectively [3].

These disorders are attributed to an assortment of factors such as substance/alcohol abuse, transport injuries, low social economic status, child abuse and the existence of chronic diseases such as Human Immunodeficiency Virus/Acquired Immune Deficiency Syndrome (HIV/AIDS), diabetes and Cerebral Vascular Accidents [4]. Among these factors, HIV is regarded as one of major factors contributing to an increase in mental disorders. According to Duffy et al. [5], people living with HIV are more than twice as likely to experience a mental health disorder.

Since its discovery in the 1980s, HIV has continued to be a major public health concern accounting for a substantial part of the global burden of disease. In Zambia, the prevalence of HIV is estimated to be 13.3 percent [6] with approximately 120,000 children ( 0 - 14 years) reported to be living with HIV by end of 2002 [7]. A substantial number (90\%) of the HIV infected adolescents acquired the infection perinatally [8]. However, recent studies indicate a decline in this fraction due to the scaled-up Prevention of Mother to Child Transmission (PMTCT) policy of testing all pregnant women for HIV, treating HIV infected with combination ART, prophylaxis ART for the newborn, avoidance of breastfeeding and the use of elective caesarean sections [9].

Before the introduction of ART in 1995, 50 percent of HIV infected children died before the age of two [10]. However, with the advent of life-prolonging antiretrovirals, HIV infection is no longer the "death sentence" in children that it was in the pre-antiretroviral era [11]. There is a marked reduction in the mortality rates among the perinatally HIV infected children. In view of this marked reduction in mortality rates, HIV has now become a chronic illness among adolescents [12]. However, new challenges are emerging in the treatment of children with HIV, including a number of risks to their psychological wellbeing. Garvie et al.'s [13] findings support this assertion and reveal an increase in the prevalence of mental health disorders among adolescents infected with HIV. Similarly, Habib et al. [14], Lowenthal et al. [15], and Mellins and Malee [16] revealed an increase in mental health problems among HIV positive adolescents.

Studies conducted by Williams et al. [17] and Mellins et al. [18] found higher than expected rates of depression, anxiety, hyperactivity, learning and other be- 
havioural problems among children born with HIV. Williams et al. [17] found that many children and adolescents with HIV present with a varied range of mental health problems. Most of the children meet clinical criteria for psychiatric disorder, particularly attention-deficit/hyperactivity disorder (ADHD). Pao et al. [19] reported that 85 percent of the youths with HIV met diagnostic criteria for at least one primary Diagnostic and Statistical Manual for Mental Disorders- $4^{\text {th }}$ Edition (DSM-IV) disorder, and 44 percent met criteria for current major depression.

Mental health problems among this population has implications for later physical health through its impact on health-related behaviour such as smoking, substance abuse, unsafe sex and non-adherence to medications. These can be detrimental to the health and well-being of HIV positive adolescents and may place others at risk for secondary HIV-transmission, treatment failure and drug resistance, which may hamper their long-term treatment and result in more significant comorbidities creating a significant public health challenge [20].

In Zambia, there has been little research into mental health of HIV positive adolescents hence the nature, prevalence and correlates of mental health problems among adolescents with HIV has not been well established. This is despite there being an increase in the number of children with HIV reaching adolescence. This paper seeks to explore the Mental Health problems experienced by HIV infected adolescents in Choma District of Zambia.

\section{Methods and Measures}

\subsection{Research Design}

A cross sectional descriptive study design was employed to obtain quantitative descriptions of mental health problems among HIV infected adolescents. To support information obtained from the adolescents and to determine some of the factors associated with mental health problems, information about mental health services was obtained from the nurses working in ART clinic and the mental health unit.

\subsection{Research Setting}

This study was undertaken at Choma General Hospital located in Choma District the provincial capital of the Southern province of Zambia. According to the CSO [6], Choma District has a population of 286,228 . The hospital at which the study took place is a second level referral hospital with a bed capacity of 204. It has all the main sub-specialties including a Mental Health Unit (the only one within the district) and ART clinic. The Mental Health Unit attends to both inpatients and outpatients staffed by eight mental health nurses and one clinical officer psychiatry. The ART clinic provides both adult and paediatric HIV/AIDS services daily except for weekends. The clinic has a cumulative total of 9552 clients on therapy of which about 2065 are adolescents [21].

This site was purposively selected as a research setting for this study because it 
was a convenient location in terms of accessibility and has a sufficient study population. Further the hospital comprises study participants whose characteristics satisfy the study requirements.

\subsection{Study Population}

For this study, the study population comprised of two groups. The first group was adolescents infected with HIV receiving treatment at the research setting plus their parents/caregivers. The second group was nurses working in the ART clinic and the mental health unit at Choma General Hospital.

\subsection{Sample Selection}

Systematic random sampling was used to select the adolescents to act as a representative sample. During this process, every $2^{\text {nd }}$ case seen at the study site between March to April 2017 was selected from the sampling frames. The sampling interval was established by dividing 160 (the accessible number of HIV positive adolescents receiving treatment at Choma General Hospital) by 103 (the sample size) giving an interval of every 2 nd person.

For the nurses, convenient sampling was used to select nurses to act as a representative sample. During this process, nurses who were available, perceived to be typical of the population and met the inclusion criteria were selected to participate in the study.

\subsubsection{Inclusion Criteria}

All HIV infected adolescents aged between 11 and 17 years receiving treatment from Choma General Hospital were eligible to participate in the study. The study included those adolescents and nurses who consented/assented to participate.

\subsubsection{Exclusion Criteria}

The study sample excluded adolescents who were physically unwell (determined clinically) because they could get tired as administration of the test battery will take some time. The study also excluded those who did not give consent or ascent to participate in the study as participation was voluntary.

\subsection{Sample Size}

In this study, a total of 103 adolescents were included in the sample. The total sample size was calculated using the prevalence formula as follows:

$$
n=\frac{z^{2} \times P \times(1-p)}{d^{2}}
$$

where $\mathrm{z}=1.96$ at $\mathrm{d}=0.05$ and $\mathrm{P}=0.24$.

The sample size required was $=\frac{1.96^{2} \times 0.24 \times(1-0.24)}{0.05^{2}}=291$

$$
\text { Final } n=\frac{n}{1+n / N}=\frac{291}{1+(291 / 160)} 0.05^{2}
$$


Plus all the nurses working in ART and mental health clinic.

\subsection{Measures}

The modified youth version of the Strengths and Difficulties Questionnaire (SDQ-Y) was used to obtain quantitative data. The SDQ-Y is a self-report, behavioural screening instrument designed to be completed by children aged $11-17$ years and is used to detect childhood emotional and behavioural problems. The tool had three sections with Section A containing demographic information, Section B had 25 items divided into five subscales comprising of five items each, generating scores for conduct problems, hyperactivity/inattention, emotional symptoms, peer problems and prosocial behaviours, while Section C had questions on stigma.

In addition to the questionnaire (self-rated SDQ) that was completed by the adolescents, the parents (guardian) were asked to complete a similar questionnaire (parent rated SDQ) though the wording was slightly different in order to have more reliable information. The researcher administered the self-rated SDQ to the adolescents who could not read or write with the help of a translated version.

Qualitative data was obtained using semi structured interview schedule. The focus of the interview was on mental health services/interventions available for the adolescents living with HIV.

\subsection{Data Collection Technique}

Before commencing the interview or administering the questionnaire, the researcher introduced himself to the study respondents and verbal permission obtained. The purpose of the study was explained and assurance of confidentiality made. When the respondents agreed to participate in the study, written consent was obtained. A Self-administered questionnaire was administered to the adolescents who completed the instruments after which they were retrieved. The researcher administered the self-rated SDQ to the adolescents who could not read or write with the help of a translated version.

To supplement information obtained from the adolescents, a semi structured interview was conducted to obtain information from the nurses working in the ART and mental health unit.

\subsection{Ethical and Cultural Considerations}

This research was granted ethical approval by the University of Zambia Biomedical Research Ethics Committee (Ref: 006-02-17) and the National Health Research Authority of Zambia (Ref: MH/101/23/10/1) before having contact with respondents. Written permission to conduct the study was obtained from Southern Province Medical Office and Choma General Hospital.

During the research process, respondents were not subjected to any physical harm as the study did not involve any invasive procedures. Potential risks that 
could have arisen from asking questions about HIV infection that might have evoked emotional reactions were explained to the participants. Prior arrangements for counselling and support were made for the anticipated emotional reactions by the participants considering the emotional nature of the topic under study. The respondents answered the questions in a comfortable environment with privacy assured and no public interference. This was done during their free time and within the shortest possible time.

The respondents were informed of the nature of the study both orally and in written so that they could make an informed decision regarding their participation. Written informed consent was obtained from all the parents/guardians of the research participants with the agreement that their children's identities would not be revealed. For this reason, pseudonyms have been used throughout. All the participating children gave their written assent before administering the questionnaire. The participants were also informed that they had the right to withdraw from the study at any time without any prejudice.

\subsection{Statistical Analysis}

Analysis of data was done using Statistical Package for Social Sciences (SPSS) version 22 and Chi square test was employed to establish the relationship between independent and dependent variables. $\mathrm{P}$ values equal or less than 0.05 were considered statistically significant. The domains of the SDQs were computed using already existing computerized algorithms for predicting mental health problems to get a total score which was then correlated with mental health problems.

Data obtained from the semi structured interview was analysed qualitatively in terms of common themes, patterns and interrelationships among the data.

\section{Results}

\subsection{Response Rates and Demographic Characteristics}

A total of 103 adolescents plus a corresponding number of parents/care givers were recruited to participate in the study. No one refused to take part, but seven responses $(6.8 \%)$ were excluded because of extensive missing data giving a useable response rate of 93.2 percent. Data about mental health services was obtained from 10 nurses working in the ART clinic and mental health unit.

In the present study, the sample included 56 girls (58.3\%) and 40 boys (41.7\%). More than half (56.3\%) of the respondents were between the ages 11 - 13 years while only 43.8 percent were between the age group $14-17$.

Most of the respondents (46\%) had attained primary level education with a small number $(6 \%)$ having attained tertiary level education. Majority of the respondents (63.5\%) lived with single parents, $24(25 \%)$ with other relatives (including grandparents) and a relatively small percentage (11\%) with both parents. 
All the respondents were HIV positive and were on antiretroviral (ARV) treatment. Most of them (98.2\%) visited the health facility at least every two months but only 24.5 percent reported that they had health problems. The majority (64\%) of the sample did not know their HIV positive serostatus.

\subsection{Prevalence of Mental Health Problems}

The findings of the current study (Figure 1) show that more than three quarters of the adolescents had mental health problems as revealed by both the Self rated SDQ (85.4\%) and the Parent rated SDQ (62.5\%). Of this number, 57.3 percent and 30.2 percent had multiple mental health problems as indicated by the self-rated and parent rated SDQ respectively.

\subsection{Mental Health Problems Experienced by Adolescents}

Figure 2 shows that 46.9 percent scored abnormal in the peer problems category according to the Self rated SDQ while the parents rated SDQ indicated that 8.3 percent of adolescents had abnormal hyperactivity scores.

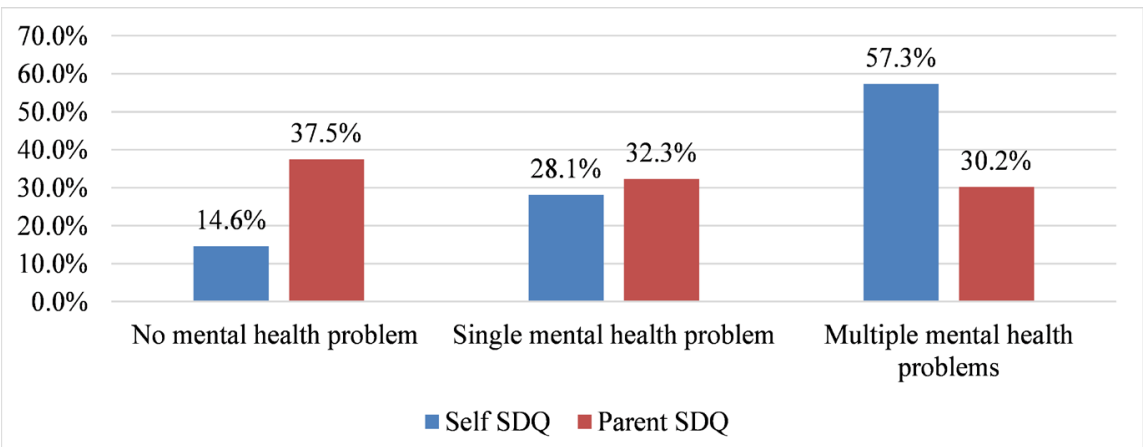

Figure 1. Prevalence of mental health problems $(n=96)$.

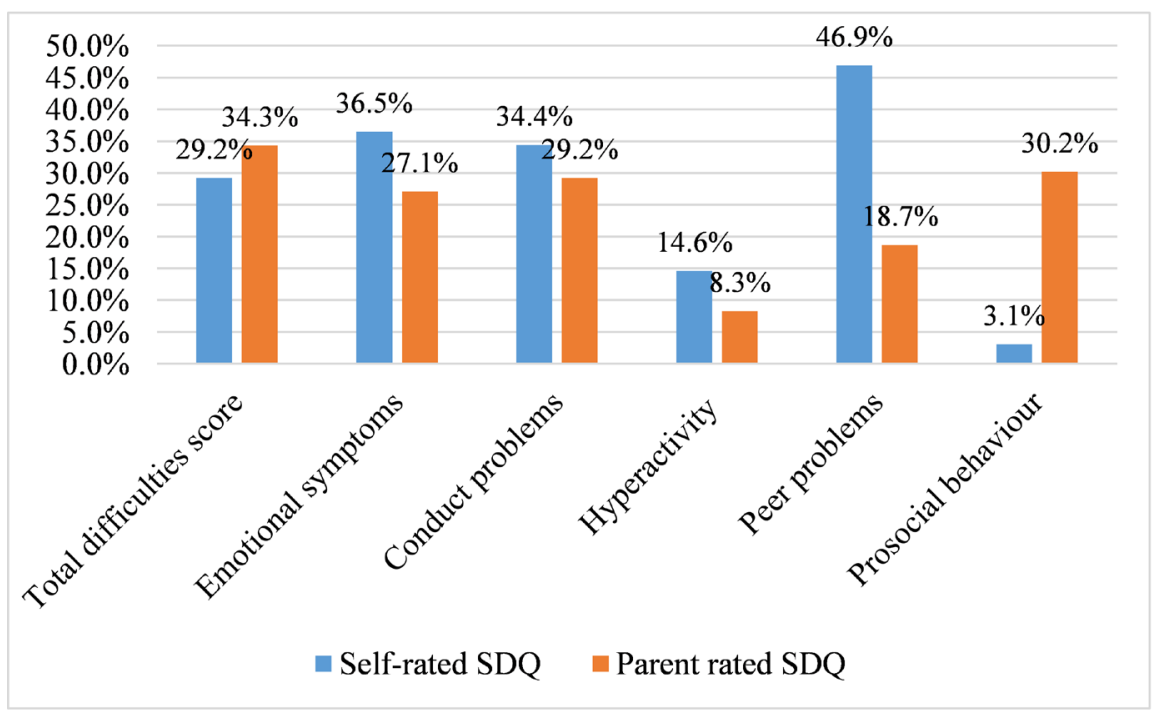

Figure 2. Self report and parent report SDQ: Abnormal range $(n=96)$. 


\subsection{Distribution of Respondents According to Levels of Internalized Stigma}

Figure 3 shows that majority of the respondents (70\%) had high level of internalized stigma.

\subsection{Stigma in Relation to Mental Health Problems}

Table 1 shows that majority (53.7\%) of the respondents who had high level of stigma had multiple mental health problems and only 10.5 percent who had moderate level of stigma had no mental health problems. Therefore, there is a statistically significant relationship between level of stigma and presence of mental health problems $\left(\mathrm{X}^{2}=1.123, \mathrm{P}=0.003\right)$.

\subsection{Services for Adolescents with HIV/AIDS}

All most all (95\%) the nurses interviewed did state that there are no specific services for these adolescents living with HIV apart from them having their own day for drug collection.

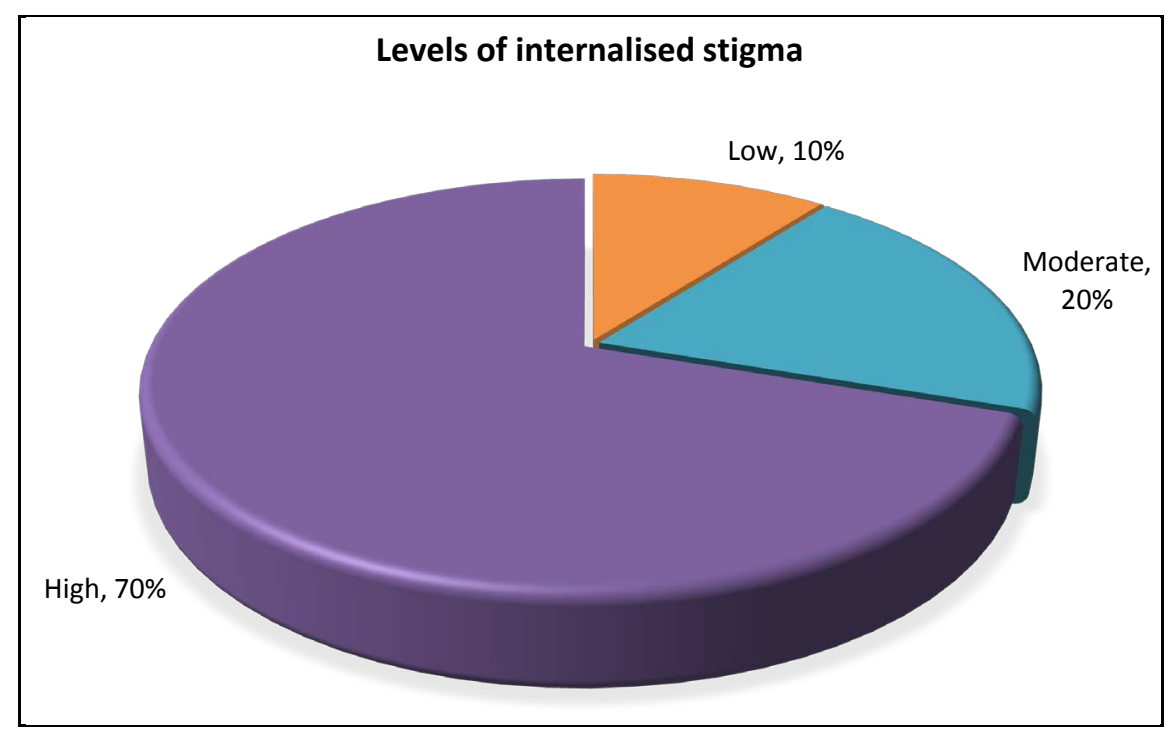

Figure 3. Levels of stigma $(\mathrm{n}=96)$.

Table 1. Stigma in relation to mental health problems.

\begin{tabular}{cccccc}
\hline & \multicolumn{3}{c}{ Mental health problem } & & \\
\cline { 2 - 4 } $\begin{array}{c}\text { Levels of } \\
\text { stigma }\end{array}$ & $\begin{array}{c}\text { No mental } \\
\text { health } \\
\text { problem }\end{array}$ & $\begin{array}{c}\text { Single mental } \\
\text { health } \\
\text { problem }\end{array}$ & $\begin{array}{c}\text { Multiple } \\
\text { mental health } \\
\text { problems }\end{array}$ & Total & P value \\
\hline Low & $2(20)$ & $2(20)$ & $6(60)$ & $10(10.4)$ & \\
Moderate & $2(10.5)$ & $4(21.1)$ & $13(68.4)$ & $19(19.8)$ & $\mathrm{X}^{2}=1.123$ \\
High & $10(14.9)$ & $21(31.3)$ & $36(53.7)$ & $67(69.8)$ & $\mathrm{P}=0.003$ \\
Total & $\mathbf{1 4}(\mathbf{1 4 . 6 )}$ & $\mathbf{2 7}(\mathbf{2 8 . 1 )}$ & $\mathbf{5 5 ( 5 7 . 3 )}$ & $\mathbf{9 6}(100)$ & \\
\hline
\end{tabular}


"The adolescents are treated like any other patient coming for treatment at this clinic. They are not a special group at all. We see them every Tuesday morning using the same facilities available to everyone. There is nothing like equipment and counselling rooms specifically for the adolescents."(Nurse 1)

"When they come for drug collection, they are counselled just like any other patients in a group by the psychosocial counsellors before their vital signs are taken in readiness for review by the clinical officer or nurse on duty." (Nurse 2)

Clients who seemed to have significant mental health problems were referred to the psychiatric clinic for screening by the clinical officer psychiatry. However most of the adolescents preferred to avoid going to the psychiatric clinic for fear of stigma and discrimination.

All the nurses indicated that assessments of the adolescents were routinely done on all the clients each time they came for reviews. Nurse one explained how the assessment of adolescents is done:

"Here in ART clinic we assess the clients firstly by checking their vital signs, then we obtain history using intake form before conducting a general physical examination. After that, we collect blood samples and send to the laboratory." (Nurse 3)

The intake form used to assess the adolescents contains demographic information, presenting symptoms, health history and diagnostic results. It mainly focuses on the physical health of the clients. Nurse four stated that:

"When assessing the adolescents, we are guided by their complaints and these are mainly physical complaints." (Nurse 4)

When asked if there was routine screening of adolescents with HIV for mental health problems, majority of the nurses stated that there were no routine or protocols followed in the screening for mental health problems among adolescents with HIV at Choma General Hospital. All participants who were interviewed agreed to having heard of some screening tools for mental health problems but could not indicate the names of the tools.

Furthermore, the study established that most nurses could not screen for and diagnose mental health problems in adolescents. This could be attributed to lack of knowledge about mental health problems. For instance, when asked about the common mental health problems experienced by adolescents, most nurses mentioned mental retardation and learning disabilities and only a few (10\%) nurses from the mental health unit mentioned conditions such as ADHD and some emotional problems.

Almost all nurses reported that psychological care was being offered to all the clients during routine reviews. However, it was noted that counselling done by the psychosocial counsellors was the only psychotherapeutic service available for the adolescents living with HIV.

"The psychosocial counsellors offer counselling to the clients on issues of adherence, disclosure of status and positive living." (Nurse 3)

When asked about their awareness of cognitive behavioural therapy (CBT) 
and other psychotherapeutic services, only six percent mentioned having heard of CBT while twenty-five percent said they had heard about other psychotherapy services such as group therapy and family therapy even though they are not competent enough to offer them. The rest of the participants showed ignorance.

The general observation was that most of the staff expressed lack of understanding of childhood mental health problems and hence had challenges screening and identifying mental health problems in the adolescents. If they suspected a child to have a mental health problem, they would refer such a child to the mental health unit for assessment and treatment. None of the health workers in the facility had some in-service trainings in mental health and psychiatry apart from the routine clinical presentations done at the hospital.

\section{Discussion of Findings}

\subsection{Mental Health Problems}

The findings of the current study (Figure 1) show that more than three quarters of the adolescents had mental health problems as revealed by both the Self rated SDQ (85.4\%) and the Parent rated SDQ (62.5\%). Of this number, 57.3 percent and 30.2 percent had multiple mental health problems as indicated by the self-rated and parent rated SDQ respectively. This demonstrates that many adolescents who participated in the study had clinically significant mental health problems.

The findings of the present study support previous research evidence [16] [22] which generally suggest that adolescents with HIV face an increased burden of mental health challenges compared with the general population. Kamau et al. [23] affirms that 48 percent of adolescents with HIV were found to have psychiatric morbidity with a significant number (25\%) of participants having more than one psychiatric disorder. Because of these problems, rates of psychiatric hospitalizations are three times higher among adolescents with HIV compared to the general population [24].

However, contrary to this assertion, Gadow et al. [11] indicates that adolescents with HIV are not at greater risk for current psychiatric problems than HIV negative peers living in similar environmental settings. Similarly, Chernoff et al. [25] found that HIV positive adolescents were no more likely to have psychiatric symptoms than age-matched HIV exposed uninfected controls.

An inquiry into the specific mental health problems experienced by adolescents with HIV showed a high prevalence of emotional and peer problems. These mental health problems mainly manifest in various distressing behaviours such as agitation, a depressed mood most of the day, a diminished ability to think or concentrate, aggression, self-harm, substance misuse and other activity that compromises sexual health. This finding is in accord with results from previous studies [23] [22] [26] indicating a high prevalence of emotional and peer problems among adolescents with HIV compared to non-infected adolescents.

Das et al. [27] reported that among the HIV positive adolescents, 45.3 percent 
suffer from psychiatric-morbid constituting emotional disorder (41.7\%), conduct disorder (37.5\%), somatization (33.3\%) and learning-disorder (29.2\%). A review of eight studies by Vreeman et al. [28] on the prevalence of psychiatric disorders amongst HIV-infected children and youth (aged 4 to 21 years) using the DSM-IV found high rates of anxiety (24\%) and depression (25\%).

On the contrary, Scharko [29] found significantly higher levels (29\%) of Attention Deficit Disorder in comparison with the other mental health problems among adolescents with HIV. Further to this Pao et al. [19] reports that the prevalence of anxiety, mood, and other behavioural disorders were the same between the HIV-exposed groups.

The discrepancy in prevalence and types of mental health problems may be attributed to methodological limitations, including small sample sizes, differences in tools used and heterogeneous definitions. Further to this, many of the studies of mental health in HIV positive adolescents conducted to date lack comparison groups and are mainly cross sectional, which makes it difficult to compare the prevalence of mental health challenges in this population to the general population. There is therefore urgent need to conduct longitudinal comparison studies to help draw conclusions about the prevalence of mental health problems and the potential contribution of HIV on the observed rates of mental health problems.

\subsection{Stigma and Mental Health Problems}

Consistent with assertions by Waluyo et al. [30] and Mupambireyi et al. [31] that stigma is very prominent among adolescents with HIV we found that majority of the respondents (69.8\%) had high level of internalized stigma.

This high prevalence of self-stigma is a source of concern because of its damaging effect on the mental wellbeing of people living with HIV by breaking down the confidence to seek mental health services.

The study also found that majority (65.4\%) of the respondents who had high level of internalised stigma had multiple mental health problems while only 14.3 percent of those had low level of internalised stigma had no mental health problem. The results suggest that internalised stigma associates significantly with mental health problems $\left(\mathrm{X}^{2}=1.123, \mathrm{P}=0.003\right)$. Therefore, stigma is a significant predictor of mental health problems among adolescents with HIV in Choma district.

These findings are consistent with Brener et al. [32] who reported that that those with HIV-related stigma had poorer psychiatric outcomes than those who were able to conceal their stigma. Further, Yi et al. [33] study, revealed that higher levels of mental disorders remained significantly associated with higher levels of stigma and discrimination in families and communities and higher levels of internal stigma. Similarly, Dow et al. [34] noted that Mental health difficulties were prevalent among HIV-positive adolescents and were associated with incomplete adherence and stigma. 
The results indicate that stigma is one of the main factors associated with mental health problems hence there is need for community-based interventions to reduce HIV stigma and address mental health among HIV-positive adolescents. This will improve not only mental health, but may also improve ART adherence and virologic suppression, improving overall health of the individual and reducing HIV risk behaviour.

\subsection{Mental Health Problems and Demographic Characteristics}

Results Table 1 showed that 27 respondents aged 11 - 23 had no mental health problems while only 10 of those aged $14-17$ had multiple mental health problems. This finding showed no statistically significant relationship between age and presence of mental health problems among adolescents with HIV in Choma District $\left(\mathrm{X}^{2}=10.847, \mathrm{P}=0.211\right)$. Therefore, age is not a significant predictor of mental health problems among adolescents with HIV in Choma District. This is supported by Malee et al. [35] who found no statistically significant age differences in relation to prevalence of psychiatric disorders at follow-up.

On the contrary, Das et al. [27] indicated that age as an independent variable is associated significantly $(\mathrm{P}<0.001)$ with psychiatric morbidity. In the same vain, Malee et al. [36] highlighted that young age was associated with Mental Health Problems among HIV positive youth. The current study finding indicates a need for large scale longitudinal study with sites systematically selected to explore the area of age in relation to mental health problems in Zambia.

In the present study, females seemed to have more mental health problems than males however the difference did not reach a level of significance $(\mathrm{P}=$ $\left.0.166, X^{2}=3.593\right)$. An analysis of the SDQ domains and gender showed no significant differences in the total SDQ score, emotional difficulty score, hyperactivity score, conduct problems and peer problems. However, boys were found to have higher scores on the prosocial scale indicating more positive social behaviours such as kindness to others.

This finding is consistent with $\mathrm{Ng}$ et al. [37] who found depression rates to be higher among HIV-infected adolescent females than males in Rwanda, but this difference was not significant. In agreement, Menon [38] in her pilot of the SDQ among Zambian adolescents reported no significant relationships between gender and any of the SDQ scores.

Nevertheless, given the lack of significant relationship between these two constructs, this assertion is rather contestable. Based on Mellins et al.'s [16] systematic review which found that, among HIV-infected youth, female sex is a risk factor for depression and anxiety, while male sex is a risk factor for behavioural problems. The inconsistent results regarding gender differences in the present study may be influenced by a small sample size and lack of representation of urban population. Therefore, this suggests a need for larger study which will be representative of both the urban and the rural population.

Results showed that majority of the respondents (63.5\%) receiving ART at 
Choma General hospital lived with single parents and a relatively small percentage (11\%) with both parents. This finding was unexpected because with the scaling up of ART, HIV related mortality has reduced significantly. Menon [22] noted that more than half of participants (55\%) with HIV lived with both parents but nearly all (97\%) lived with at least one family member. The differences could be related to the differences in the study settings with Menon's study having been conducted in Lusaka city while this study was conducted in Choma a typical peri urban area with most of the respondents coming from the surrounding villages.

An investigation to determine possible relationship between who the respondents lived with and presence of mental health problems reviewed no statistically significant relationship $\left(\mathrm{X}^{2}=1.509, \mathrm{P}=0.825\right)$. This finding is contrary to Menon et al. [38] who noted that children who did not live with a family member had higher scores on the hyperactivity subscale $(Z=2.01, P=0.04)$ although parental loss was not related to the child's mental health. A previous study by Lester et al. [39] had also found that bereavement was not associated with higher rates of psychologic disorder and it may be that other factors, such as quality of foster care, may be more important determinants of psychologic outcomes.

\subsection{Mental Health Services for Adolescents with HIV}

The findings have shown a deficit in the provision of mental health services to adolescents with HIV in Choma district, confirming what Strasser and Gibbons [40] found in selected Districts of Zambia. We found that there are no dedicated health services for adolescents living with HIV hence subjecting them to the same services accessed by the general population. Such services however may not categorically address needs and preferences of this growing population. When asked if there was routine screening of adolescents with HIV for mental health problems, majority of the nurses stated that there were no routine or protocols followed in the screening for mental health problems among adolescents with HIV at Choma General Hospital. The impression created from such a response was that there is limited attention to the provision of mental health services to adolescents with HIV. This makes cases to remain undetected and untreated. This is in line with Mellins and Malee [16] who reported that mental health problems are common among adolescents with HIV yet are often undiagnosed and may become severe or chronic if unrecognized and untreated.

Lack of age appropriate services which consider the needs and preferences of this growing population for the disclosure of HIV status to adolescents or for any other common mental disorders such as depression and anxiety maybe a contributing factor to the increase in the number of adolescents with mental health problems.

Severe shortages in mental health workers were noted to be one of the main barriers to the effective provision of mental health services for adolescents with HIV. For instance, there is no qualified Psychiatrist or even a clinical psycholo- 
gist within the district. The critical shortage of Health-care staff has also been reported by Fabri et al. [41]. According to them, this shortage makes mental health care access for this population in sub-Saharan Africa inadequate. For the participants in the present study, mental health needs are supposed to be met by the one Clinical Officer Psychiatry plus the six Registered Mental Health nurses. However, these health workers are primarily found within the psychiatric unit hence it is difficult for the adolescents with HIV to benefit from their services as the psychiatric unit is highly stigmatized hence a good number of adolescents shun it.

In view of the severe shortages in mental health workers, the study advocates for a task-shifting approach which typically includes the training of lay community members in the delivery of basic psychosocial care such as cognitive behavioural therapy and problem-solving therapy. This task-shifting approach and the use of adolescent HIV-positive peer educators in offering peer-based psychosocial support could help reduce the mental health problems among adolescents.

The study also found that most nurses expressed lack of understanding of mental health problems among adolescents with HIV. Because of the lack of knowledge, nurses had challenges screening, identifying and managing mental health problems among adolescents with HIV. In addition, almost all nurses expressed lack of awareness of CBT and other Psychotherapeutic services. This is in line with Tumwesigye and Abate [42] who argues that a limited number of health care workers have adequate knowledge and skills to comfortably practice paediatric HIV care with respect to pre-test and post-test counseling, disclosure of HIV to children or helping care takers to disclose HIV status to children provide. The lack of knowledge about mental health issues is a hindering factor to the improvement of the health status of adolescents with HIV.

This finding further is in line with what was reported by Earnshaw et al. [43] who stated that poor approach to treatment of psychological conditions could be attributed to the situation that health systems are over-stretched and under-resourced, and patients together with health institutions have a variety of competing issues which are not limited to mental health but also to other medical and surgical conditions.

\section{Conclusions}

Several conclusions can be drawn from this study, the most important being adolescents with HIV are a high-risk population to mental health problems. A considerable proportion of this population has multiple mental health problems indicative of significant impairment. In addition, adolescents with high levels of internalized stigma are more likely to have multiple mental health problems. With regards to mental health service provision, there is a significant gap which is because of lack of training in mental health for nurses attending to these adolescents. 
Drawing from the present study findings which have revealed that mental health problems are prevalent among adolescents with HIV yet are most often undiagnosed by the nurses, providing adequate continued professional development related to mental health is essential.

\section{Strengths and Limitations of the Study}

The major strength of the study is that it was the first one of its kind to explore the mental health problems experienced by HIV positive adolescents in Choma District and the relatively good response rate. However, the small number of participants in this study limits the generalization of the outcome. Further, resources could not allow the study to be conducted on a large scale because the project was not adequately funded and therefore, the site was conveniently selected. This means that the results may not be representative of the larger population.

\section{Disclosure Statement}

No potential conflict of interest was reported by the authors.

\section{Acknowledgements}

I wish to express deep gratitude to the University of Zambia through the Norwegian Programme for Capacity Development in Higher Education and Research for Development (NORHED Project) for the financial support without which the project would not have been possible.

\section{References}

[1] WHO (2010) World Health Organisation Human Resources Report. World Health Organisation, Geneva, Switzerland.

[2] Uys, L. and Middletons, L. (2008) Mental Health Nursing: A South African Perspective. 5th Edition, Juta and Co. Ltd., Cape Town, South Africa.

[3] $\mathrm{MOH}$ (2005) Mental Health Policy, Lusaka. Zambia.

[4] Cassels, M. (2013) Mental Disorders Leading Cause of Nonfatal Illness Worldwide. http://www.Medscape.Com/viewarticle/810132

[5] Duffy, M., Sharer, M., Cornman, H., Pearson, J., Pitorak, H. and Fullem, A. (2017) Integrating Mental Health and HIV Services in Zimbabwean Communities: A Nurse and Community-Led Approach to Reach the Most Vulnerable. Journal of the Association of Nurses in AIDS Care, 28, 186-198. http://doi.org/10.1016/j.jana.2015.09.003

[6] CSO, MOH and ICF International (2014) Zambia Demographic and Health Survey 2013-14. Central Statistical Office, Ministry of Health, and ICF International, Rockville, Maryland, USA.

[7] CDC (2014) Centres for Disease Control HIV/AIDS Report, Lusaka, Zambia.

[8] National Aids Council (2015) Zambia Country Report.

[9] Mburu, G., Ram, M., Oxenham, D., Haamujompa, C., Iorpenda, K. and Ferguson, L. (2014) Responding to Adolescents Living with HIV in Zambia: A Social-Ecological Approach. Children and Youth Services Review, 45, 9-17. 
http://doi.org/10.1016/j.childyouth.2014.03.03

[10] Laughton, B., Cornell, M., Boivin, M. and Van Rie, A. (2013) Neurodevelopment in Perinatally HIV-Infected Children: A Concern for Adolescence. Journal of the International AIDS Society, 16, Article ID: 18603.

[11] Gadow, K.D., Chernoff, M., Williams, P.L., Brouwers, P., Morse, E., Heston, J., Nachman, S., et al. (2010) Co-Occuring Psychiatric Symptoms in Children Perinatally Infected With HIV and Peer Comparison Sample. Journal of Developmental Behaviour Paediatrics, 31, 116-128.

[12] Busza, J., Besana, G.V.R., Mapunda, P. and Oliveras, E. (2014) Meeting the Needs of Adolescents Living with HIV through Home Based Care: Lessons Learned from Tanzania. Children and Youth Services Review, 45, 137-142.

[13] Garvie, P.A., Bret, Z., Malee, K., Nichols, S., Smith, R., Wilkins, M. and Williams, P.L. (2014) Discordance of Cognitive and Academic Achievement Outcomes in Youth with Perinatal HIV Exposure. Paediatrics Infectious Diseases Journal, 33, 232-238.

[14] Habib, A.G., Yakasai, A.M., Owolabi, L.F., Ibrahim, A., Habib, Z.G., Gudaji, M., Nashabaru, I., et al. (2013) Neurocognitive Impairment in HIV-1-Infected Adults in Sub-Saharan Africa: A Systematic Review and Meta-Analysis. International Journal of Infectious Diseases, 17, e820-e831. http://doi.org/10.1016/j.ijid.2013.06.011

[15] Lowenthal, E.D., Bakeera-Kitaka, S., Marukutira, T., Chapman, J., Goldrath, K. and Ferrand, R.A. (2014) Perinatally Acquired HIV Infection in Adolescents from Sub-Saharan Africa: A Review of Emerging Challenges. Lancet Infectious Diseases, 14, 627-639. http://doi.org/10.1016/s1473-3099(13)70363-3

[16] Mellins, C.A. and Malee, K.M. (2013) Understanding the Mental Health of Youth Living with Perinatal HIV Infection: Lessons Learned and Current Challenges. Journal of the International AIDS Society, 16, Article ID: 18593.

[17] Williams, P., Chernoff, M., Angelidou, K., Brouwers, P., Kacanek, D., Deygoo, N., Gadow, K., et al. (2013) Participation and Retention of Youth with Perinatal HIV Infection in Mental Health Research Studies: The IMPAACT P1055 Psychiatric Comorbidity Study. Journal of Acquired Immune Deficiency Syndrome, 63, 401-409. https://doi.org/10.1097/QAI.0b013e318293ad53

[18] Mellins, C.A., Elkington, K.S., Leu, C.-S., Santamaria, K.E., Dolezal, C., Wiznia, A., Abrams, E.J., et al. (2012) Prevalence and Change in Psychiatric Disorders among Perinatally HIV-Infected and HIV-Exposed Youth. AIDS Care, 24, 953-962. https://doi.org/10.1080/09540121.2012.668174

[19] Pao, M., Lyon, M., D’Angelo, J., Schuman, W.B. and Tipnis, T. (2000) Psychiatric Diagnoses in Adolescents Seropositive for the Human Immunodeficiency Virus. Archives of Pediatrics and Adolescent Medicine, 154, 240-244. https://doi.org/10.1001/archpedi.154.3.240

[20] Mellins, C.A., Brackis-Cott, E., Leu, C., Elkington, K.S., Dolezal, C., Wiznia, A., Abrams, E.J., et al. (2009) Rates and Types of Psychiatric Disorders in Perinatally Human Immunodeficiency Virus-Infected Youth and Seroreverters. Journal of Child Psychology Psychiatry, 50, 1131-1138.

https://doi.org/10.1111/j.1469-7610.2009.02069.x

[21] CGH (2015) Choma General Hospital Action Plan.

[22] Menon, A., Glazebrook, C. and Ngoma, M.S. (2009) Mental Health of HIV Positive Adolescents in Zambia. Medical Journal of Zambia, 36, 151-156.

[23] Kamau, J.W., Kuria, W., Mathai, M., Atwoli, L. and Kangethe, R. (2012) Psychiatric 
Morbidity among HIV-Infected Children and Adolescents in a Resource-Poor Kenyan Urban Community. AIDS Care, 24, 836-842. https://doi.org/10.1080/09540121.2011.644234

[24] Gaughan, D.M., Hughes, D.M., Oleske, M., Malee, K., Gore C. and Nachman,S. (2004) Psychiatric Hospitalizations among Children and Youths with Human Immunodeficiency Virus Infection. Pediatrics, 113, e544-51. https://doi.org/10.1542/peds.113.6.e544

[25] Chernoff, M., Nachman, S., Williams, P., Brouwers, P., Heston, J. and Hodge, J. (2009) Mental Health Treatment Patterns in Perinatally HIV-Infected Youth and Controls. Pediatrics, 124, 627-636. https://doi.org/10.1542/peds.2008-2441

[26] Vreeman, R., McCoy, B. and Lee, S. (2017) Mental Health Challenges among Adolescents Living with HIV. Journal of the International AIDS Society, 20, Article ID: 21497.

[27] Das, P.K., Maiti, S. and Sinha, N.K. (2016) Abundance of Psychiatric Morbidity in Perinatally HIV Infected Children and Adolescents with Comparison to Their HIV Negative Sibling. Neurology, Psychiatry and Brain Research, 22, 167-172.

[28] Vreeman, R.C., Scanlon, M.L., McHenry, M.S. and Nyandiko, W.M. (2015) The Physical and Psychological Effects of HIV Infection and Its Treatment on Perinatally HIV-Infected Children. Journal of the International AIDS Society, 18, Article ID: 20258. https://doi.org/10.7448/IAS.18.7.20258

[29] Scharko, A.M. (2006) DSM Psychiatric Disorders in the Context of Pediatric HIV/AIDS. AIDS Care, 18, 441-445. https://doi.org/10.1080/09540120500213487

[30] Waluyo, A., Culbert, G.J., Levy, J. and Norr, K. (2015) Understanding HIV-Related Stigma among Indonesian Nurses. Journal of the Association of Nurses in AIDS Care, 26, 69-80. https://doi.org/10.1016/j.jana.2014.03.001

[31] Mupambireyi, Z., Bernays, S., Bwakura-Dangarembizi, M. and Cowan, F.M. (2014) "I Don't Feel Shy because I will Be among Others Who Are Just Like Me...": The Role of Support Groups for Children Perinatally Infected with HIV in Zimbabwe. Children and Youth Services Review, 45, 106-113. https://doi.org/10.1016/j.childyouth.2014.03.026

[32] Brener, L., Callander, D., Slavin, S. and de Wit, J. (2013) Experiences of HIV Stigma: The Role of Visible Symptoms, HIV Centrality and Community Attachment for People Living with HIV. AIDS Care, 25, 1166-1173. https://doi.org/10.1080/09540121.2012.752784

[33] Yi, S., Chhoun, P., Suong, S., Thin, K., Brody, C. and Tuot, S. (2015) AIDS-Related Stigma and Mental Disorders among People Living with HIV: A Cross-Sectional Study in Cambodia. PLOS ONE, 10, e0121461. https://doi.org/10.1371/journal.pone.0121461

[34] Dow, D.E., Turner, E.L., Shayo, A.M., Mmbaga, B., Cunningham, C.K. and O’Donnell, K. (2016) Evaluating Mental Health Difficulties and Associated Outcomes among HIV-Positive Adolescents in Tanzania. AIDS Care, 3, 825-833. https://doi.org/10.1080/09540121.2016.1139043

[35] Mellins, C.A., Tassiopoulos, K., Malee, K., Moscicki, A.-B., Patton, D., Smith, R., Seage, G.R., et al. (2011) Behavioral Health Risks in Perinatally HIV-Exposed Youth: Co-Occurrence of Sexual and Drug Use Behavior, Mental Health Problems, and Nonadherence to Antiretroviral Treatment. AIDS Patient Care and STDs, 25, 413-422. https://doi.org/10.1089/apc.2011.0025

[36] Malee, K.M., Tassiopoulos, K., Siberry, G., Williams, P.L., Hazra, R., Smith, R.A., Mellins, C.A., et al. (2011) Mental Health Functioning among Children and Ado- 
lescents with Perinatal HIV Infection and Perinatal HIV Exposure. AIDS Care, 23, 1533-1544. https://doi.org/10.1080/09540121.2011.575120

[37] Ng, L.C., Ahishakiye, N., Miller, D.E. and Meyerowitz, B.E. (2015) Life after Genocide: Mental Health, Education, and Social Support of Orphaned Survivors. International Perspectives in Psychology, 4, 83-97.

[38] Menon, J.A. (2014) The Strengths and Difficulties Questionnaire: A Pilot Study on the Reliability and Validity of the Self-Report Version to Measure the Mental Health of Zambian Adolescents. Journal of Health Science, 2, 127-134.

[39] Lester, P., Chesney, M., Cooke, M., Weiss, R., Whalley, P. and Perez, B. (2002) When the Time Comes to Talk About HIV: Factors Associated with Diagnostic Disclosure and Emotional Distress in HIV-Infected Children. Journal of Acquired Immune Deficiency Syndromes 31, 309-317. https://doi.org/10.1097/00126334-200211010-00006

[40] Strasser, S. and Gibbons, S. (2014) The Development of HIV-Related Mental Health and Psychosocial Services for Children and Adolescents in Zambia: The Case for Learning by Doing. Children and Youth Services Review, 45, 150-157. https://doi.org/10.1016/j.childyouth.2014.03.032

[41] Fabri, M., Ingabire, C., Cohen, M., Donenberg, G. and Nsanzimana, S. (2015) The Mental Health of HIV-Positive Adolescents. The Lancet Psychiatry, 8, e21. https://doi.org/10.1016/S2215-0366(15)00291-6

[42] Tumwesigye, N. and Abate, C. (2008) Psychosocial Care and Counseling for HIV Infected Children and Adolescents: A Training Curriculum. Regional Centre for Quality Health Care (RCQHC), ANECCA, CRS, Kampala, Uganda.

[43] Earnshaw, V.A., Smith, L.R., Chaudoir, S.R., Amico, K.R. and Copenhaver, M.M. (2013) HIV Stigma Mechanisms and Well-Being among PLWH: A Test of the HIV Stigma Framework. AIDS and Behavior, 17, 1785-1795. https://doi.org/10.1007/s10461-013-0437-9 